\title{
Self-assembly of anionic, ligand-coated nanoparticles in lipid membranes
}

DOI:

10.1039/c6nr05853a

\section{Document Version}

Accepted author manuscript

Link to publication record in Manchester Research Explorer

\section{Citation for published version (APA):}

Angelikopoulos, P., Sarkisov, L., Cournia, Z., \& Gkeka, P. (2017). Self-assembly of anionic, ligand-coated nanoparticles in lipid membranes. Nanoscale, 9(3), 1040-1048. https://doi.org/10.1039/c6nr05853a

\section{Published in:}

Nanoscale

\section{Citing this paper}

Please note that where the full-text provided on Manchester Research Explorer is the Author Accepted Manuscript or Proof version this may differ from the final Published version. If citing, it is advised that you check and use the publisher's definitive version.

\section{General rights}

Copyright and moral rights for the publications made accessible in the Research Explorer are retained by the authors and/or other copyright owners and it is a condition of accessing publications that users recognise and abide by the legal requirements associated with these rights.

\section{Takedown policy}

If you believe that this document breaches copyright please refer to the University of Manchester's Takedown Procedures [http://man.ac.uk/04Y6Bo] or contact uml.scholarlycommunications@manchester.ac.uk providing relevant details, so we can investigate your claim.

\section{OPEN ACCESS}




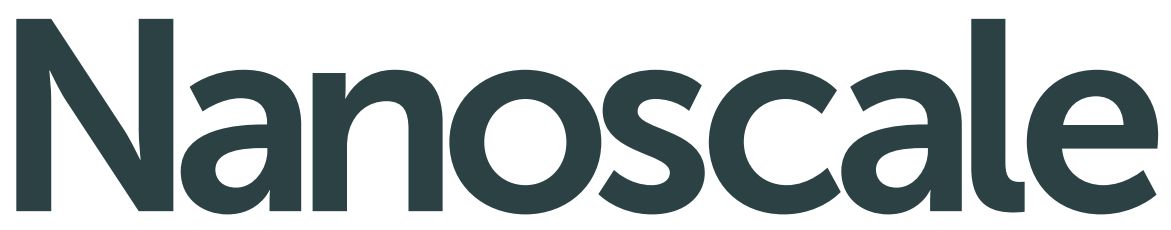

rsc.li/nanoscale

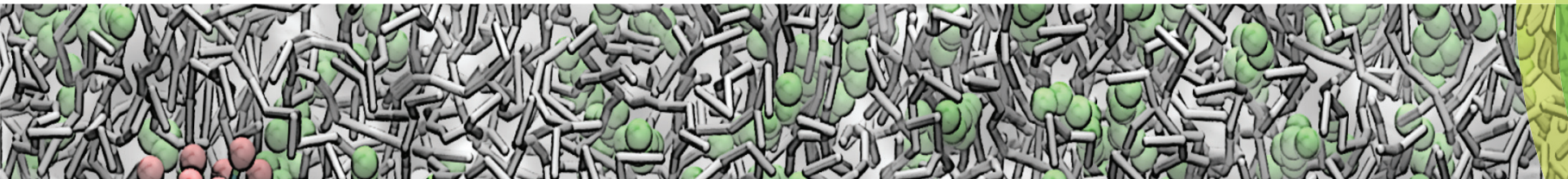

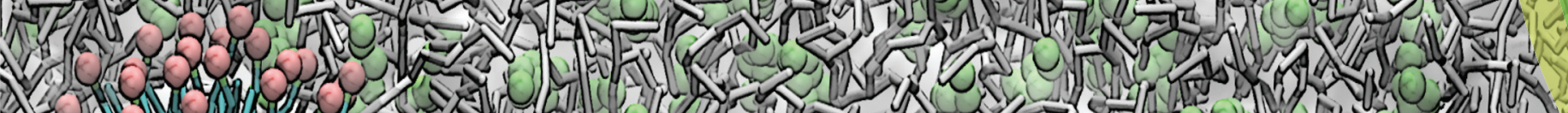
(5)

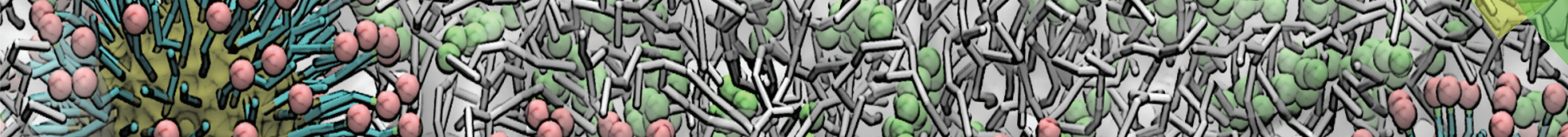

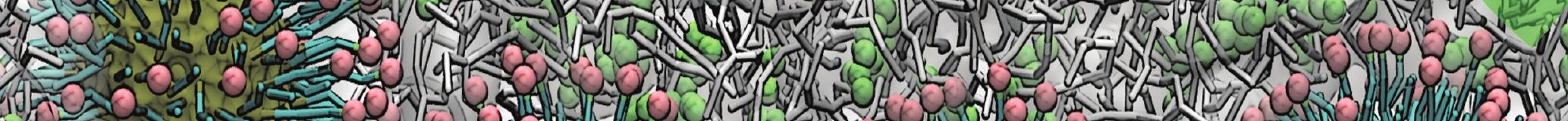
14. 15 (5) 1. $x$ (5)

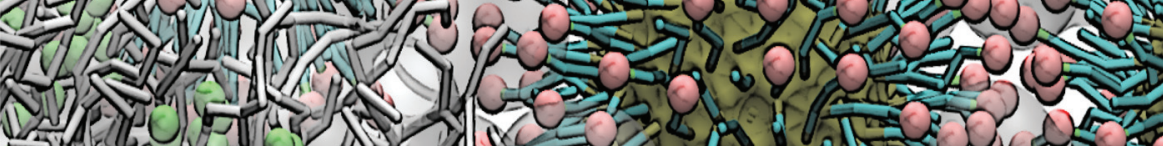
15.1. -2 15(5)

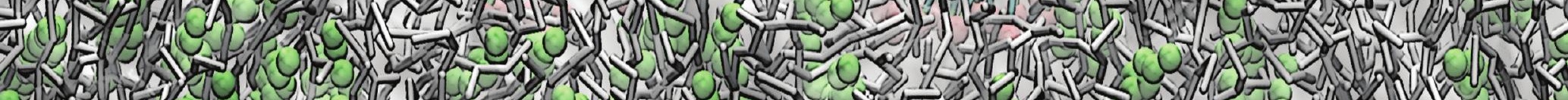

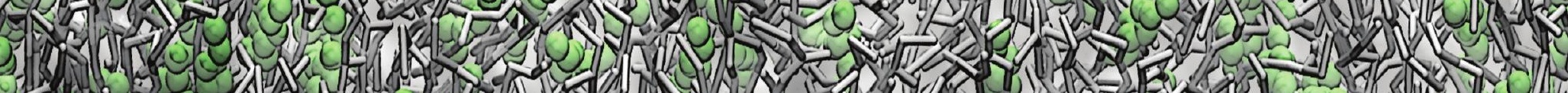

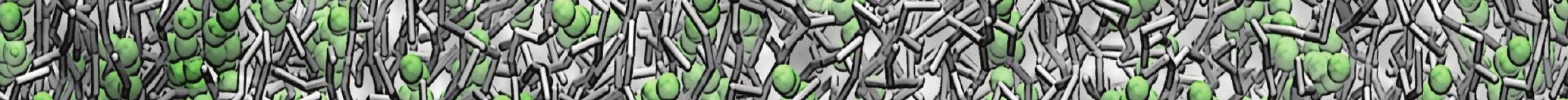
(1)

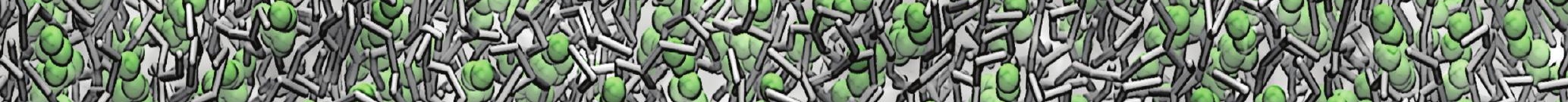
Q 


\title{
Nanoscale
}

\section{PAPER}

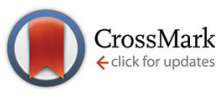

Cite this: Nanoscale, 2017, 9, 1040

\section{Self-assembly of anionic, ligand-coated nanoparticles in lipid membranes $\uparrow$}

\author{
Panagiotis Angelikopoulos, $\uparrow^{\mathrm{a}}$ Lev Sarkisov, ${ }^{\mathrm{b}}$ Zoe Courniac and Paraskevi Gkeka* $\S^{\mathrm{c}}$
}

Ligand-functionalized nanoparticles (NPs) are a promising platform for imaging and drug delivery applications. A number of recent molecular simulation and theoretical studies explored how these NPs interact with model lipid membranes. However, interactions between ligand-coated NPs leading to possible cooperative effects and association have not been investigated. In this coarse-grained molecular dynamics study, we focus on a specific case of several anionic, ligand-coated NPs embedded in a lipid membrane. Several new effects are observed. Specifically, in the presence of cholesterol in the membrane, NPs tend to form linear clusters, or chains. Analysis of the driving forces for this association reveals an important role of the recently discovered orderphobic effect, although we acknowledge that a combination of factors must be at play. At the same time, we argue that the specific linear shape of the clusters is a result of a subtle balance between the forces that stabilize a NP in the membrane and the forces that drive the NP-NP association processes. These effects, observed for the first time in the NP-membrane systems, have also direct correspondence to similar effects in protein-membrane systems and these links between the two realms should be explored further.

Received 24th July 2016, Accepted 7th October 2016

DOI: $10.1039 / c 6 n r 05853 a$ www.rsc.org/nanoscale
Here, we focus on a particular class of NPs, developed by Stellacci and co-workers. ${ }^{11-13}$ A NP from this class consists of a gold core and is coated with hydrophobic (1-octanethiol, OT) and hydrophilic (11-mercapto-1-undecanesulphonate, MUS) ligands, carrying a negative charge on their termini. At specific compositions, binary mixtures of these ligands self-assemble on the NP surface into regular, well-defined patterns, i.e. stripes. Previously, NPs of 4.3 to $4.9 \mathrm{~nm}$ core diameter with surface featuring striped patterns of hydrophobic and hydrophilic areas were shown to be able to translocate across the dendritic cell membrane through an energy and receptor independent mechanism. ${ }^{13}$

Computational studies of these systems, emerging from several independent research groups, have drawn the following picture of the interaction between anionic, ligand-coated NPs and model membranes, represented as lipid bilayers. The first key point is that the flexibility of the ligands is a crucial property of these NPs. In an aqueous solution, this flexibility of ligands leads to their re-arrangement on the NP surface, driven by both the minimization of the contact area between hydrophobic groups and water and the surface charge re-distribution. As a result, the underlying pattern of the ligand attachment to the surface of the NP is hidden under the termini group pattern formed as a response to the local environment. ${ }^{14}$

A non-disruptive fusion of the NP with the model cell membrane is initiated by a protrusion of one of the ligand chains into the bilayer, a process assisted by interactions between the charged ligand termini and the polar lipid head groups. ${ }^{15}$ On 
a longer timescale, the NP can eventually reposition into the bilayer core. For this to happen, the charged termini of individual ligands must cross from one leaflet of the bilayer to the opposite leaflet, a process that is repeated for several surface ligands until the NP is inside the membrane core. ${ }^{16}$ This process is associated with substantial energy barriers and requires long simulation times to be observed (tens of microseconds according to Simonelli et al.). ${ }^{16}$

Once inside the lipid bilayer, a NP is stabilized in this configuration by a ligand snorkelling effect. ${ }^{15,17}$ This effect describes the tendency of the charged termini of the ligands to avoid the hydrophobic core of the bilayer and "snorkel" to its surface, where they form contacts with the charged lipid head groups and water. It is important to recognize, of course, that the details of these processes, including the propensity of the NP to interact with and partition into the membrane and the stability of the snorkelling configuration, depend on the size of the NP, ligand length and chemistry, as well as other factors.

Now, with the possibility of the anionic, ligand-coated NPs to partition into the bilayer established, a question naturally arises whether the already inserted NPs influence the propensity of other NPs to enter and cross the membranes. Before this question can be addressed it is important to understand how the embedded NPs affect the structural and dynamical properties of the membranes and how they interact with each other. This is the issue addressed in this article.

Our original research objective was quite simple: to investigate the structure and component distribution of dipalmitoylphosphatidylcholine (DPPC) bilayers containing cholesterol in the presence of two or more ligand-decorated, anionic NPs (these properties for a single NP embedded in a DPPC bilayer with different concentrations of cholesterol have been reported previously). ${ }^{17}$

Interestingly, what we observe is a persistent association of NPs with each other, leading to dimers, trimers and tetramers forming in the system depending on the number of present NPs. Given the substantial amount of literature on association of other membrane inclusions, such as membrane proteins, this result may not seem that surprising. As has been shown in our previous publication, a NP inclusion changes the lipid bilayer structural properties in its vicinity: the bilayer is thinner and more disordered in the vicinity of a NP; and if the system contains cholesterol, the concentration of cholesterol is lower in the vicinity of the NP compared to the bulk bilayer. ${ }^{17}$ As a result of the latter effect, although the bulk bilayer may be in the liquid-ordered phase (typical for systems with cholesterol present at physiological temperatures), the vicinity of the NP tends to have liquid-like characteristics. The local cholesterol depletion and disordering of the bilayer caused by the $\mathrm{NP}$, resembles the nucleation of a disordered domain in the vicinity of membrane proteins. ${ }^{18}$ Existence of the disordered domains around protein inclusions within ordered lipid bilayers has been recently shown to drive protein self-assembly via the so-called orderphobic effect. ${ }^{18}$ In the case of the specific NPs under consideration herein, snorkelling of the surface ligands may also lead to local membrane deformation, depending on the degree of the mismatch between the size of the NP and ligands and the thickness of the bilayer. These factors combined (membrane thinning and deformation) are responsible for areas of high surface tension in the membrane and, similarly to the membrane proteins with different degree of hydrophobic mismatch, ${ }^{18}$ this could lead to the self-assembly of the NPs, in a process aimed to reduce the area of high surface tension. Other effects can also contribute to the NP self-assembly. Similarly to what has been reported for membrane proteins, another factor could be the Helfrich's entropic undulation force amongst NP inclusions that, in the case of membrane proteins, leads to thermal membrane undulations, which in turn influence the effective interactions between the proteins and the membrane. ${ }^{19}$ Depletion attraction, an effective interaction well known in the colloidal systems ${ }^{20,21}$ is another force that could contribute to the re-organization of NPs within the lipid bilayer.

So far our observations establish a clear analogy between the processes behind membrane protein self-assembly and the behaviour of NPs embedded in a lipid bilayer. An interesting and original effect observed in this study, however, is the tendency of NPs to always form chains, as opposed to other structures or clusters. As we argue in this article, this is associated with a subtle balance between the forces that keep the NPs inside the membrane (ligands snorkelling to the surface and anchoring the NPs) and the forces driving the NP self-assembly. Upon the self-assembly, some of the ligands are not able to snorkel to the bilayer-water interface anymore. The number of these ligands is proportional to the area of the NP surface participating in the formation of contacts with another NP. We show that if a NP has, colloquially speaking, too many contacts, there are simply not enough ligands snorkelled to the surface to keep it embedded in the bilayer and the particular spatial arrangement of the NPs becomes unstable. In the conclusion section, we discuss the implications of these findings.

\section{Results and discussion}

\section{Dimer formation by anionic, ligand-coated NPs}

Our first case study considers unbiased CG Molecular Dynamics (MD) simulations with two NPs embedded in a DPPC lipid bilayer containing either $0 \mathrm{~mol} \%$ or $30 \mathrm{~mol} \%$ cholesterol (see Table S1 in the ESI $\dagger$ for the complete list of simulations and the corresponding simulation times and Fig. S1 $\uparrow$ for the model of NP).

In the $30 \mathrm{~mol} \%$ cholesterol system, the two NPs form a dimer on the timescale of few microseconds, with Fig. 1a showing a typical configuration of the system and Fig. S2 and $\mathrm{S} 3 \uparrow$ showing the evolution of the system upon the dimerization process. There are several structural features of the dimer to note. Sodium ions, shown in orange, associate with the negatively charged ligand termini; furthermore, some CG water particles are present in the contact area between NPs. This conformation could potentially lead to membrane leakage due to 

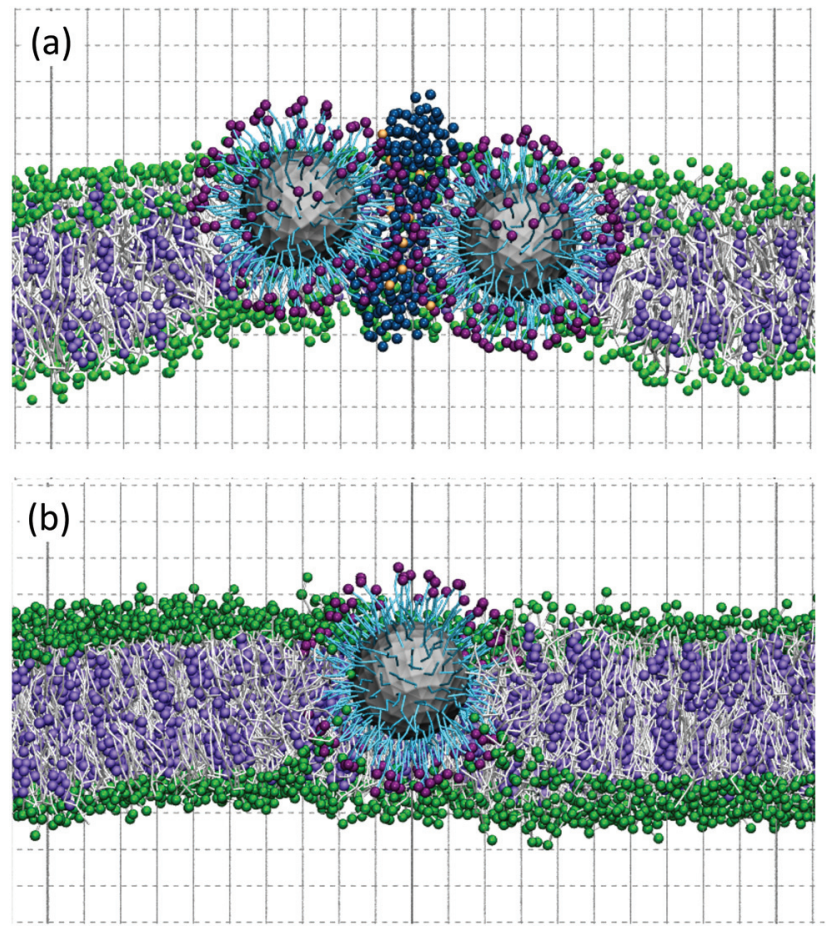

Fig. 1 Dimer formation in a lipid bilayer containing 30 mol\% cholesterol. (a) The snorkelling effect. Water and ions at a distance within $5 \mathrm{~nm}$ from both NPs are shown in blue and orange, respectively, while bulk water is not shown for clarity. Lipid tails are shown in white licorice representation. DPPC lipid heads ( $\mathrm{NC3}$ and PO4 groups) are shown as green particles, the NP core is shown as grey surface, NP ligand hydrophobic tails are shown in licorice representation colored in cyan, polar NP ligand termini are shown as purple particles. Cholesterol is represented as mauve particles. (b) A toroidal-shaped lipid organization. Coloring in (b) is the same as in (a). The two snapshots correspond to the two perspectives of the $x y$-plane.

pore formation as has been previously reported. ${ }^{22}$ From a different perspective (Fig. 1b) the lipid bilayer interface assumes a toroidal structure in the vicinity of the complex. Snorkelling of the ligands is observed in the areas of the NPS that are not in contact with the water/ion layer between them. The NP dimer is stable until the end of the simulation, i.e. $12 \mu \mathrm{s}$, with the inter-NP distance being approximately $6 \mathrm{~nm}$ between the NP centers of mass. In contrast to the cholesterolcontaining system, in the cholesterol-free lipid bilayer no dimer formation is observed on the timescale of the simulation performed in the present study (up to $14.6 \mu \mathrm{s}$ ). In Fig. S4, $\dagger$ we present the time series of the distances between the centers of mass of the NP for the two types of lipid bilayers.

To quantitatively assess the thermodynamic stability of the dimer observed by the unbiased MD simulations within the cholesterol-containing lipid bilayer and to potentially identify the effect of cholesterol on the dimerization process, we calculate the interaction free energy, represented by the Potential of Mean Force (PMF), as a function of NP-NP distance, using the umbrella sampling technique. ${ }^{23,24}$ The PMF profiles for the two NPs in $0 \mathrm{~mol} \%$ and $30 \mathrm{~mol} \%$ cholesterol systems are

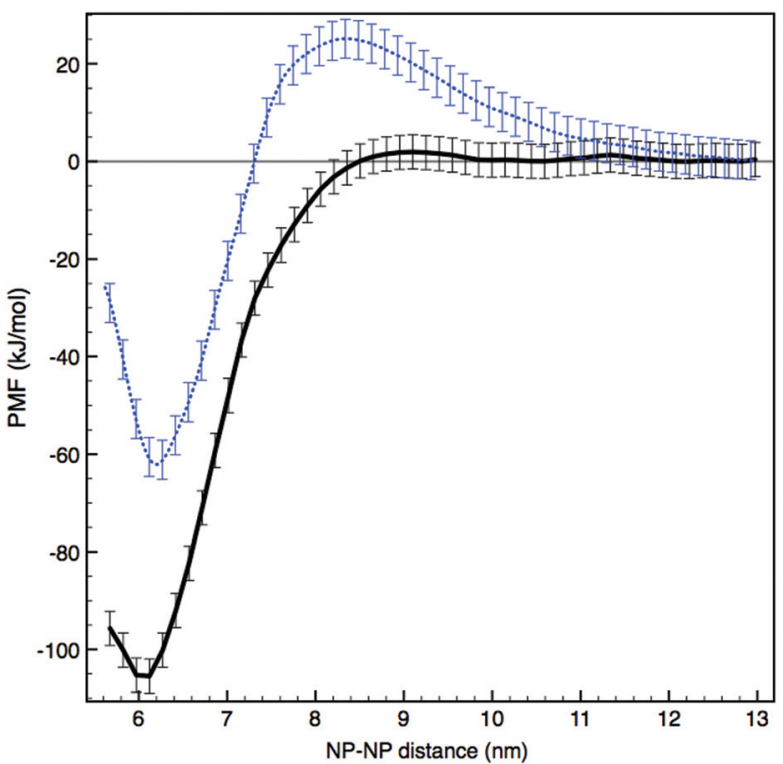

Fig. 2 Potential of mean force as a function of NP-NP distance. The blue line corresponds to the cholesterol-free case and the black line corresponds to the cholesterol-containing lipid bilayer.

shown in Fig. 2. The free-energy minimum for a NP approaching another in the $30 \mathrm{~mol} \%$ cholesterol bilayer is approximately $-100 \mathrm{~kJ} \mathrm{~mol}^{-1}$ and is located at $6 \mathrm{~nm}$ separation distance between the centers of the NPs, in agreement with the unbiased simulation. The PMF levels out at a $\sim 8.5 \mathrm{~nm}$ distance between the NPs. In contrast to this picture, in the cholesterol-free lipid bilayer, we observe a region of unfavourable interactions between 7 and $12 \mathrm{~nm}$ of a separation distance between the NPs, beyond which the NPs behave as free and independent particles. The presence of a $20 \mathrm{~kJ} \mathrm{~mol}^{-1}$ energy barrier in the cholesterol-free membrane could be a potential explanation of why NP dimerization is not observed in this system.

The free-energy minimum for a NP approaching another in the cholesterol-free membrane is approximately $-60 \mathrm{~kJ} \mathrm{~mol}^{-1}$ and it is also located at a $6 \mathrm{~nm}$ inter-NP distance. This implies that once the free energy barrier is overcome, the two NPs must form a dimer in the cholesterol free system. To probe the possibility and stability of this dimer, we perform an unbiased MD simulation in the cholesterol-free system, starting with two NPs at a $6 \mathrm{~nm}$ distance from each other. In a simulation exceeding $6.0 \mu \mathrm{s}$, the dimer remains intact, with many structural characteristics being common with the dimer formed in the cholesterol-containing bilayer: water particles and sodium ions are present in the contact region while snorkelling is also evident on the surface of the NPs that is not in contact with water.

The question however remains as to why a $20 \mathrm{~kJ} \mathrm{~mol}^{-1}$ energy barrier is present in the cholesterol-free system and not in the $30 \mathrm{~mol} \%$ cholesterol system. In the following sections we will try to identify the factors that contribute to these two different situations. 


\section{Molecular picture of the membrane-mediated association processes in a system with several NPs}

Intrigued by the dimerization of the anionic, ligand-coated NPs in the cholesterol containing membrane and our PMF results, we extend our studies to systems with more than two NP inclusions. We employ unbiased CG MD simulations with three and four NPs in two different types of lipid bilayers, again one containing $0 \mathrm{~mol} \%$ and one containing $30 \mathrm{~mol} \%$ cholesterol (Table S1 $\dagger$ ). In Fig. S5-S7, $\dagger$ we present the time series of the distances between the centers of mass of the NPs. As in the case of the two NPs, clusters are observed in all the systems with cholesterol, but not in any of the cholesterol-free systems.

To illustrate the underlying details of the formation of clusters in the $30 \mathrm{~mol} \%$-cholesterol system, we will focus on a specific case with four NPs embedded in the bilayer. As shown in Fig. $\mathrm{S} 7, \uparrow$ in this system a dimer forms at about $7.5 \mu \mathrm{s}$, followed by the formation of a trimer at $15 \mu$ s and finally by the formation of a tetramer at $17.7 \mu \mathrm{s}$, which remains stable until the end of the simulation at $19.4 \mu \mathrm{s}$. Additional visualization of the evolution of this system is provided in the ESI in Fig. S8. $\dagger$

From our previous study we expect cholesterol to avoid being in the direct vicinity of NPs. ${ }^{17}$ Indeed, our calculations support this picture and reveal formation of low cholesterol areas around NPs and high cholesterol content areas elsewhere (Fig. 3). Cholesterol depletion in our previous work was also accompanied by an increase in the disorder of lipids in the vicinity of the NP. In Fig. 4 we demonstrate that the same effect takes place in the multi-NP system as well. A length mismatch between the bilayer hydrophobic core and the hydrophobic ligands on the surface of the NP-inclusion is also

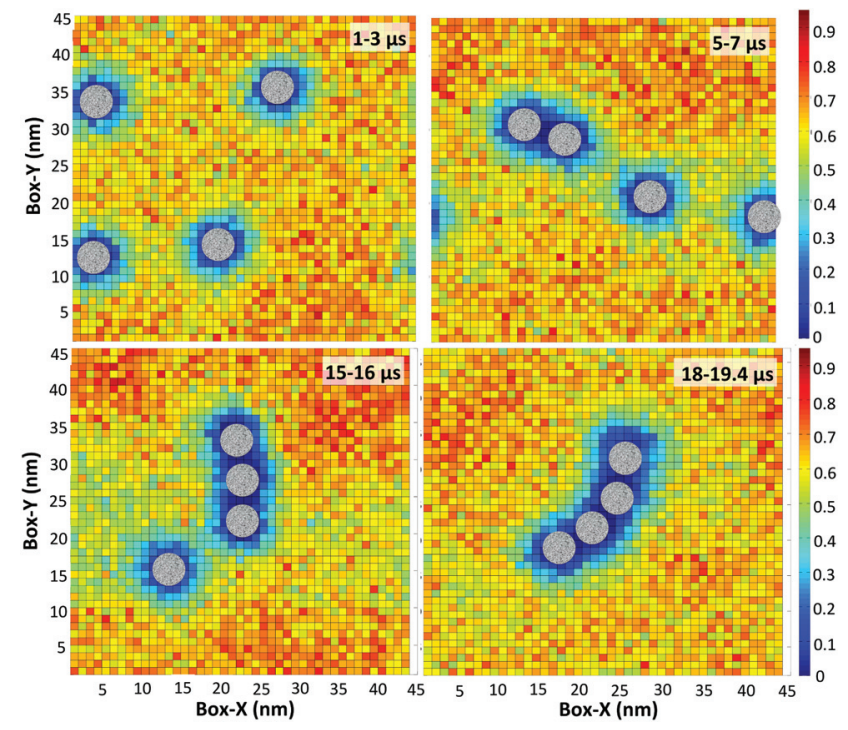

Fig. 3 Evolution of cholesterol density of the lipid bilayer containing $30 \mathrm{~mol} \%$ cholesterol and four NP inclusions, which form a linear tetramer. Density units are $\mathrm{nm}^{-3}$.
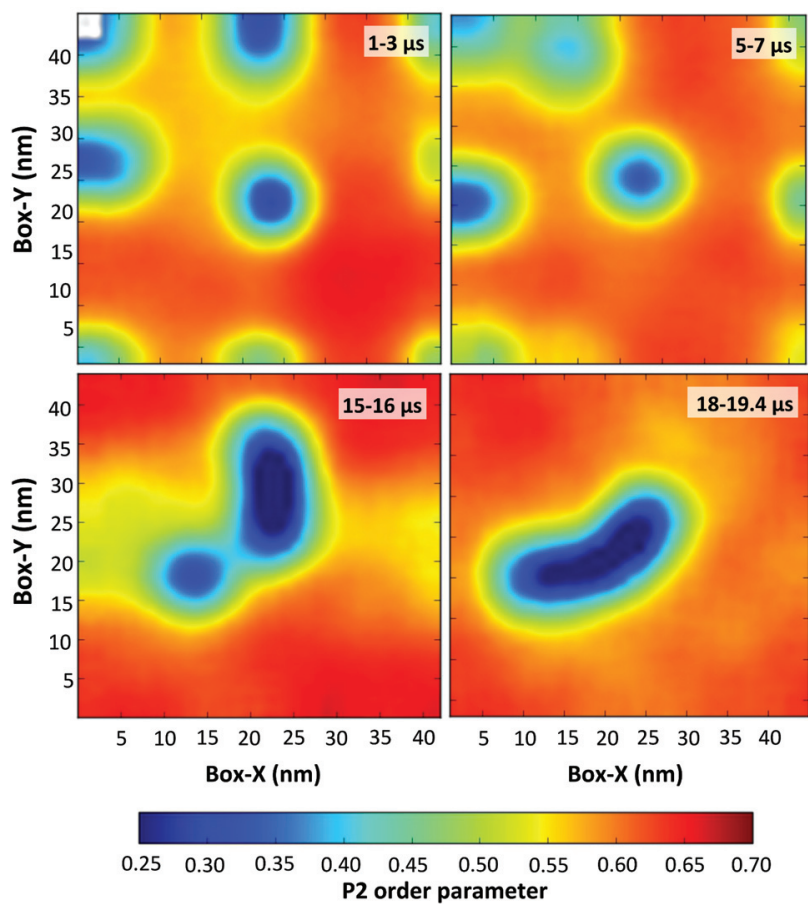

Fig. 4 Evolution of lipid order parameters in the lipid bilayer containing $30 \mathrm{~mol} \%$ cholesterol and four NP inclusions, which form a linear tetramer.

observed and is directly linked with a local perturbation in the lipid packing in the vicinity of the NP inclusion (a snapshot of the system is shown in Fig. S9†). In general, the hydrophobic mismatch is defined as the difference between the hydrophobic length of the membrane inclusion and the hydrophobic thickness of the membrane (herein it is defined as the average distance between PO4 CG groups of the opposite bilayer leaflets). The distortion of the lipid bilayer in the vicinity of a ligand-coated NP is caused by several factors. The size of the NP and the length of the hydrophobic ligands determine the range of the values for the hydrophobic thickness of the membrane to which the NP can adapt. Also, as can be seen from Fig. $S 9, \dagger$ the charged termini of the hydrophilic ligands associate with the head groups of the lipids (snorkelling effect), and, depending on the ligand position and size, may tend to pull the lipid head groups towards the center of the bilayer, further reducing its thickness in the vicinity of the NP. In all these processes the flexibility and length of the ligands as well as the core size of the NP are crucial. In Fig. S10, $\dagger$ we present the evolution of the radial lipid bilayer thickness during the association of NPs, which indicates a local thinning in the vicinity of the anionic NPs, in agreement with our previous work. ${ }^{17}$ Overall, our analyses taken together indicate that the NPs presence leads to cholesterol depletion, decrease in lipid ordering as well as decrease in lipid bilayer thickness in the vicinity of the NPs.

With this picture in mind let us now return to the issue of the differences between cholesterol-free and $30 \mathrm{~mol} \%$ cholesterol systems. For this, below we discuss possible driving 
forces for the NP association and how these forces depend on the presence of cholesterol.

As this scenario has been extensively covered in protein studies, our first investigation focuses on the mechanism, where membrane inclusions self-assemble to reduce areas of high surface tension, caused by the hydrophobic mismatch between the inclusion and the hydrophobic thickness of the membrane. As the thickness of the membrane varies with cholesterol concentration, the hydrophobic mismatch and, consequently, all the driving forces emerging as a result of this mismatch are also a function of cholesterol concentration. To explore this driving force in the $30 \mathrm{~mol} \%$ cholesterol system, we calculate the evolution of the area associated with lower bilayer thickness compared to the bulk values (see details in the methodology). As shown in Fig. 5, the area associated with the lower bilayer thickness actually increases as the simulation progresses, before the final dip in the last few microseconds of the simulation. This contradicts the expected trend if the driving force was trying to minimize the high surface tension area. We also note that this property is sensitive to the thickness threshold value (that is the value of the bilayer thickness at a certain distance from the center of NP, beyond which the bilayer is considered to be in the bulk state, unaffected by the NP presence), as shown in the same figure, and based on the obtained results it is hard to make any conclusive remarks.

Recently, another scenario for transmembrane protein selfassembly has been proposed. ${ }^{18}$ This scenario considers a lipid bilayer in an ordered phase and a protein inclusion with a hydrophobic thickness matching the hydrophobic thickness of the disordered membrane phase. As a result, this leads to a

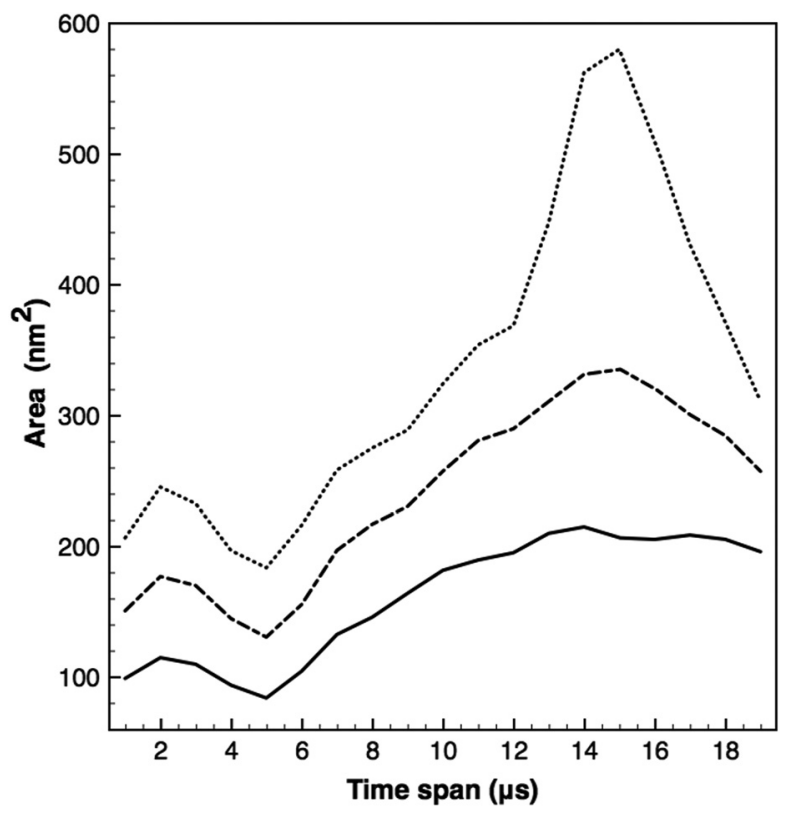

Fig. 5 Area of the membrane region with thickness lower than a particular threshold. Dotted, dashed and full lines correspond to thresholds of $39.5,40.5,41.5 \AA$, respectively. The bulk value of bilayer thickness for $30 \mathrm{~mol} \%$ cholesterol is $43.5 \AA$. region of the disordered structure around the protein inclusion and the formation of an interface between ordered and disordered regions of the membrane. According to the proposed mechanism, protein inclusions associate with each other to reduce the net interfacial free energy. The authors argue that this effect is mediated by the proximity to the ordered-disordered phase transition in the membrane. We speculate that a similar effect should take place in the cholesterol-containing system as the bulk lipid bilayer tends to be in the liquid-ordered phase (with $30 \mathrm{~mol} \%$ cholesterol), while in the direct vicinity of the NP, there is a substantial disorder in the lipid bilayer structure. To identify regions that are in the liquid-ordered phase we employ the well-established lipid order parameter metric (see Methods). Fig. 6 shows the evolution of the length of the ordered-disordered interface as a function of time. In the same figure we also show the evolution of the area associated with the disordered domain. Interestingly, while the length of the interface boundary is decreasing, the area of the disordered domain is increasing. In the ESI $\dagger$ we provide a geometric argument on how this situation can arise.

A complementary property to the analysis above is the distribution of cholesterol within the system. As has been already discussed, we observe depletion of cholesterol in the vicinity of the NPs and this effect is coupled to the structural features of the lipid bilayer near NPs and in the bulk bilayer. Here, we calculate the evolution of the interface between domains of low and high cholesterol concentrations. This calculation depends on the threshold value of the cholesterol concentration, which

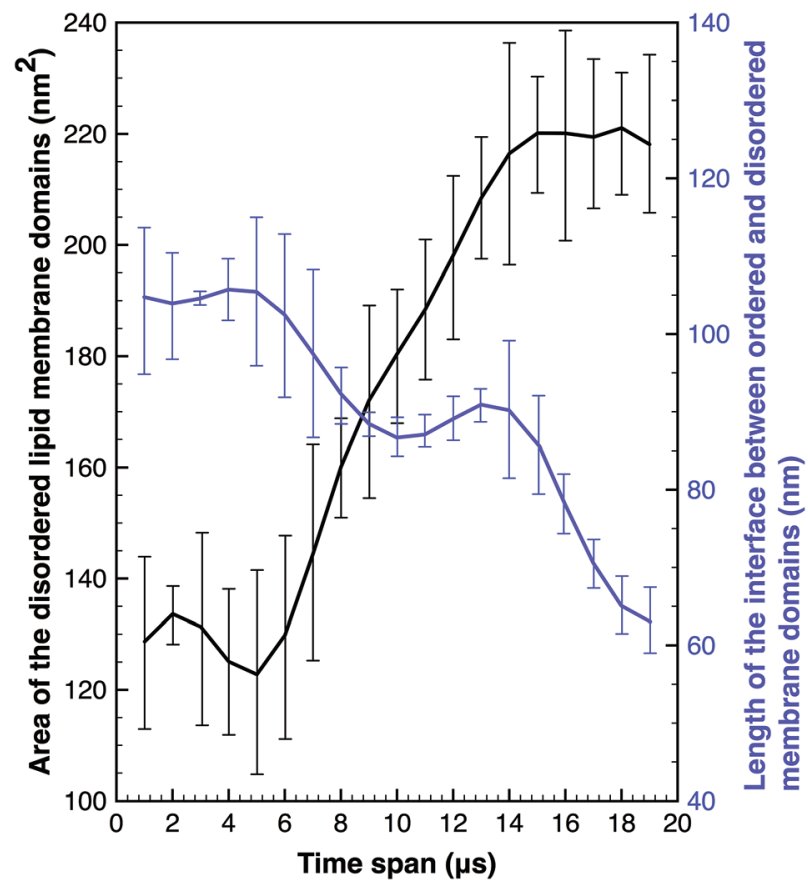

Fig. 6 Evolution of ordered-disordered lipid bilayer interface (blue) and surface (black) of the disordered area during the tetramer formation. The error bars represent the standard deviation taken over 200 ns block samples during the course of each $1 \mu$ s averaging. 


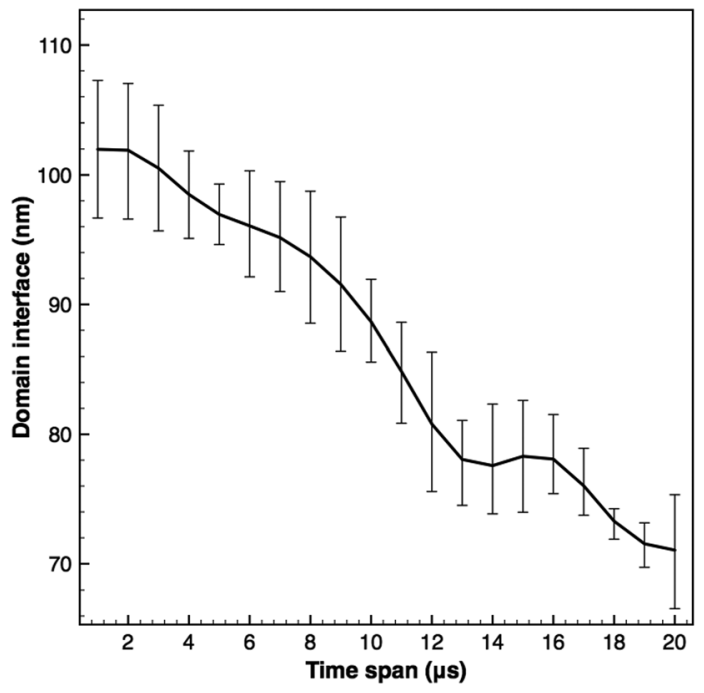

Fig. 7 Evolution of low-high cholesterol interface during the tetramer formation.

separates the regions of high and low cholesterol concentration. Here, we choose the value of $0.3 \mathrm{~nm}^{-3}$, which is less than $1 \%$ of the bulk cholesterol concentration value, although other values have been explored all pointing to a qualitatively similar picture. In Fig. 7, we present the evolution of low-bulk cholesterol interface during the tetramer formation. At the beginning, when each NP appears as a monomer in the system, the domain interface fluctuates at around $105 \mathrm{~nm}$. During the process of self-assembly, this value is decreased until the formation of the tetramer, when it reaches the value of $70 \mathrm{~nm}$. The trend shown in Fig. 7 indicates a tendency of minimization of the interface between cholesterol depleted areas and bulk membrane. Given that higher cholesterol concentration is also associated with the bilayer being in the lipid-ordered phase and a higher bilayer thickness leading to greater hydrophobic mismatch, this result is hardly surprising and is consistent with the previous observations based on the direct detection of the liquid-ordered regions using order parameter.

The presence of cholesterol may also contribute to the depletion forces between the NPs. In this case, the lack of cholesterol between the NPs and the presence of cholesterol around them causes effective attraction between them. This process could be similar to the depletion effects observed for protein and colloidal systems and may be responsible for lowering the free energy barrier in the system that includes cholesterol. ${ }^{21}$ Analysis of the cholesterol concentration presented above allows us to visualize depletion of cholesterol in between the NPs upon their association. An estimate of the possible depletion effects between the two NPs, based on the simplified Asakura-Oosawa theory, ${ }^{20}$ gives an additional $7-18 \mathrm{~kJ} \mathrm{~mol}^{-1}$ contribution to the lowering of the free energy of association in the $30 \mathrm{~mol} \%$ cholesterol compared to the cholesterol-free system. We also note that the depletion forces are relatively short range $(\sim 5 \mathrm{~nm})$, which corresponds to the location of the minimum in the PMF.
Finally, we note that intrinsic as well as NP-induced lateral bilayer heterogeneity implies changes in the macroscopic bilayer properties as well as changes in the way the bilayer mediates the interaction and organization of NPs. One of the properties that modulate effective inclusion interactions is membrane bending rigidity. Bending rigidity is significantly increased upon cholesterol inclusion in a lipid bilayer, ${ }^{25,26}$ which in turn leads to smaller membrane thermal fluctuations. This could be another factor responsible for the differences in the effective NP interactions in the cholesterol-free systems and systems containing cholesterol.

\section{Geometry of NP clusters}

What is particularly interesting about the NP self-assembly process shown in Fig. 3 is the formation of chain-like structures. Originally thought as a mere curiosity and an odd result of a particular simulation run, this picture persistently occurred in simulations with different initial conditions. A complete set of simulations summarized in the Table S1 $\dagger$ suggests that the formation of chains in these systems is not accidental but rather it is a stable configuration of the system, prompting us to investigate a possible origin of this behaviour. Firstly, the optimal solution to the interface minimization problem is not the formation of linear clusters but the formation of clover-like shapes or triangular topologies similar to those observed previously for membrane proteins. ${ }^{27}$ Consequently, if the dominant factor affecting the NP association was only the orderphobic effect, the observed clusters would not be linear but clover-like. It can be thus concluded that either more than one factors are responsible for the linear association of anionic NPs in cholesterol-containing membranes or what we observe is an intermediate transient cluster formation. To investigate further these possibilities we will follow a reverse engineering reasoning.

We focus on the hypothesis that the observed linear clusters are not in the global energy minimum (that is, in equilibrium state) but correspond to transient states, which will eventually reform into clover-like structures. We start by setting up preformed clusters of three and four NPs positioned in a triangular and square formation, respectively, in a cholesterol-containing lipid bilayer. These preformed clusters are then subjected to unbiased MD simulations. In Fig. S16, $\dagger$ we present the evolution of the non-linear tetramer. The tetramer is constructed in a way that maintains the features of the stable NP dimer, i.e. water lining between the NPs and snorkelling of the ligand tails that interact with the lipid bilayer (Fig. S16, $\dagger t=0 \mu \mathrm{s}$ ). Eventually $(\sim 6 \mu \mathrm{s})$, the NPs come to a closer distance until a significant deformation of the membrane occurs, which leads to two of the NPs being pushed away from the midplane of the membrane $(\sim 10 \mu \mathrm{s})$. Finally, one of these two NPs is expelled almost completely out of the lipid bilayer while another is also significantly shifted towards the water phase (Fig. S16 and $\left.\mathrm{S} 17^{\dagger}\right)$ ). A similar behaviour is observed in the non-linear trimer with two NPs being pushed away from the bilayer midplane (Fig. S18 and S19†). 
The instability of the non-linear clusters can be explained if we consider the mechanism that stabilizes the specific anionic NP in a lipid bilayer, i.e. the snorkelling of the anionic ligand termini of the NP, which leads to their interaction with the polar head groups of the lipids as well as with water and ions. However, in the case of NP association, several of these anionic groups are also needed to stabilize the inter-NP interface (Fig. 1). Consequently, if too many of the ligand polar termini of the NP are involved in the formation of the NP-NP interface, there is, colloquially speaking, nothing to bond the NP to the bilayer and thus it slides out of the bilayer core to the surface.

Interestingly, formation of chain clusters has been recently observed in coarse-grained molecular dynamics simulations of gramicidin A (gA) protein inclusions in several types of lipid bilayers. The authors argued that this is a result of a balance between the forces that drive the proteins together (surface tension, depletion forces, etc.) and forces that stabilize the protein inclusion in the membrane (extent of contact between the hydrophobic amino acids and bilayer core). ${ }^{27}$

\section{Methods}

\section{Systems description}

The two membranes considered in the present study are described as a single-component lipid bilayer (pure 1,2-dipalmitoyl-sn-glycero-3-phosphocholine (DPPC) bilayer) or a twocomponent lipid bilayer using the MARTINI CG model for DPPC, cholesterol, and water. ${ }^{28}$ The MARTINI model is also employed for the construction of the NP using an approach previously introduced by us. ${ }^{14,17}$ Shortly, the NP considered in the present study has a core diameter of $3 \mathrm{~nm}$ coated with regular, striped patterns of hydrophobic (octanethiol, OT) and hydrophilic, negatively charged (11-mercapto-1-undecanesulphonate, MUS) domains in a 1:1 MUS : OT ratio (Fig. S1 $\dagger$ ). Additional details on the structure and properties of the NP are provided in the ESI. $\uparrow$ The CG simulations presented in this article were performed with the GROMACS simulation package, version 4.6.6. ${ }^{29}$ The cholesterol-free lipid bilayer is comprised of $\sim 8000$ DPPC lipid molecules, while the lipid bilayer with $30 \mathrm{~mol} \%$. cholesterol concentration is comprised of $\sim 5000$ DPPC lipid and 2000 cholesterol molecules. The bilayers were simulated in excess of water, with approximately 100 water molecules per lipid (or, equivalently, 28 CG water molecules per lipid), a ratio well above the degree of hydration observed in multilamellar vesicles for fluid (Ld) bilayers and the even less hydrated gel (Lg) bilayers. ${ }^{30}$ Both systems were previously constructed by us. ${ }^{17}$ Two, three and four NPs are placed in the bilayer using VMD. ${ }^{31}$ The final system sizes were approximately $45 \mathrm{~nm} \times 45 \mathrm{~nm} \times 15 \mathrm{~nm}$.

\section{Simulation parameters}

MD simulations were performed with constant pressure, temperature, and number of particles (NPT ensemble). ${ }^{29}$ The temperature was kept constant at $323 \mathrm{~K}$ using the Berendsen thermostat with a relaxation time of $1.25 \mathrm{ps}^{32}$ The pressure of the system was semi-isotropically coupled and maintained at 1 bar using the Berendsen algorithm with a time constant of $0.22 \mathrm{ps}$ and a compressibility of $3 \times 10^{-5} \mathrm{bar}^{-1}$. The non-bonded potential energy functions were cut off and shifted at $12 \AA$ smoothly decaying between 9 and $12 \AA$ for van der Waals forces and throughout the whole interaction range for the treatment of electrostatic forces. MD simulations were performed using a 25 fs integration time step.

\section{Potential of mean force calculations}

The Potential of Mean Force (PMF) as a function of the distance between two NPs embedded in a lipid bilayer was calculated using the Umbrella Sampling protocol of GROMACS for a cholesterol-free membrane and a membrane containing $30 \mathrm{~mol} \%$ cholesterol concentration. For the calculations, the position of one of the NPs was fixed. The initial distance between the fixed NP and the second NP was $13 \mathrm{~nm}$. The final (closest) NP-NP distance was $5.6 \mathrm{~nm}$ and the whole NP-NP distance was divided into 64 windows. In each window, a different initial configuration was set up with the NP placed at the corresponding distance from the center of mass of the fixed NP. Then, the biasing potential was applied in order to restrain the NP at the given position, and the system was left to equilibrate at the restrained position for $200 \mathrm{~ns}$. Subsequently, the system was simulated for another $400 \mathrm{~ns}$, with the biasing potential applied to restrain the center of mass of the NP at a required distance from the fixed NP. The NP was left free to rotate around its restrained center of mass. A single PMF profile required a total simulation time of $38.4 \mu \mathrm{s}$. A force constant of $750 \mathrm{~kJ} \mathrm{~mol}^{-1} \mathrm{~nm}^{-2}$ was applied, following the approach by Gkeka et al. ${ }^{17}$ In order to obtain the unbiased PMFs, we used the weighted histogram analysis method (WHAM) ${ }^{23}$ with 200 bins and a tolerance of $10^{-7}$ for the convergence of WHAM equations.

\section{Analysis}

The thickness of the bilayer was calculated from the difference between the average distance from all phosphate groups in the two leaflets. The second-rank order parameter $P 2=\langle 1 / 2$ $\left.\left(3 \cos ^{2} \theta-1\right)\right\rangle$ was computed for consecutive bonds with $\theta$ being the angle between the direction of the bond and lipid bilayer normal. The APL@Voro program where Voronoi diagrams and Delaunay triangulations are generated for the different lipids in the system as well as for any inclusions considered was used for Fig. S8. $\uparrow^{33}$ A combination of GROMACS Tools, ${ }^{29} \mathrm{VMD}^{31}$ and in-house scripts was used for the rest of the analysis presented herein.

\section{Conclusions}

The original motivation for this study was to explore the structure of model lipid membranes in the presence of several embedded anionic, ligand-coated NPs representing the NPs developed by Stellacci and co-workers. Following our original work on a single NP, the current study confirms that in the 
vicinity of these NPs the lipid bilayer has lower thickness, lower cholesterol concentration and lower order compared to the bulk liquid-ordered bilayer. However, in addition to this structural analysis we also discovered several interesting effects.

In $30 \mathrm{~mol} \%$ cholesterol bilayer these NPs tend to associate in linear clusters, however no cluster formation was observed in cholesterol-free membranes. Preliminary calculations using the Arrhenius formula indicate that the $20 \mathrm{~kJ} \mathrm{~mol}^{-1}$ barrier of NP-NP association observed in the PMF calculations presented herein for the cholesterol-free systems should be crossed on the time scale of our simulations. The fact that spontaneous formation of clusters has not been observed in any of our runs for cholesterol-free system may indicate that we somehow underestimate this barrier and other factors are at play. This is to be further investigated in subsequent studies.

The presented analysis identifies the orderphobic effect, which drives protein self-assembly in disordered domains around protein inclusions within ordered lipid bilayers, as the most probable driving force for the NP self-assembly in the $30 \mathrm{~mol} \%$ cholesterol system; this also serves as a plausible explanation why spontaneous self-assembly of NPs is not observed in the cholesterol-free system.

Within the clusters, NPs have a layer of water and sodium cations between them and are stabilized within the bilayer via the ligand snorkelling effect. Since the formation of a contact between the NPs inhibits some of the ligands ability to snorkel, only certain number NP contacts can be formed before the assembly becomes unstable. We believe it is this subtle balance of the forces driving NPs together and the forces that stabilize them inside the bilayers that is responsible for the linear geometry of the NP clusters.

This behaviour is contrasted by considering several purely hydrophobic NPs, featuring no ligands and represented as spheres, embedded in the lipid bilayer containing cholesterol. Our preliminary studies indicate that these NPs accumulate at the interface between the cholesterol rich, ordered domains and cholesterol-lean, liquid domains, acting essentially as lineactants (agents which reduce line tension between domains). Importantly, they do not form clusters analogous to the ligand-decorated NPs. This highlights the important role of the charged ligands in the NP self-assembly process, although the impact of the type, length, concentration of ligands and other parameters on the process still require further investigation.

Water domains between the NPs, their transport characteristics, implications of this effect on cytotoxicity of NPs and their drug-delivery potential, as well as how this effect can be modulated and controlled by engineering NP architecture and ligands, are phenomena and processes that need to be investigated in future studies.

What makes the observations presented herein particularly interesting is the direct link with the recent analogous phenomena reported for membrane proteins, including the orderphobic effect as the driving force for protein self-assembly and formation of protein clusters. In principle, the order- phobic effect can be exploited in the assembly of nano-objects within lipid bilayers into more complex architectures. Overall, the similarities between natural membrane proteins and manmade nano-objects offer a range of technological possibilities to be further investigated and exploited.

\section{Acknowledgements}

We acknowledge PRACE for awarding access to the computational facility CURIE at GENCI@CEA, France. PG was partly funded by the Reference Framework (NSRF) 2011-2013, National Action "Cooperation", under grant entitled "Magnetic Nanoparticles for targeted MRI therapy (NANOTHER)", with code "11SYN-1-1799". The programme is cofunded by the European Regional Development Fund and national resources. LS would also like to acknowledge Prof. Davide Marenduzzo for the helpful comments on depletion effects and AsakuraOosawa theory. PG would like to thank Dr Franck Augé and Dr Andreas Karlsson who assisted in the proof-reading of the manuscript.

\section{Notes and references}

1 R. Fanciullino, J. Ciccolini and G. Milano, Crit. Rev. Oncol. Hematol., 2013, 88, 504-513.

2 S. C. Coelho, M. C. Pereira, A. Juzeniene, P. Juzenas and M. A. N. Coelho, J. Controlled Release, 2015, 213, 152-167.

3 M. A. Lauzon, A. Daviau, B. Marcos and N. Faucheux, J. Controlled Release, 2015, 206, 187-205.

4 H. M. Ding and Y. Q. Ma, Small, 2015, 11, 1055-1071.

5 S. Pogodin, M. Werner, J. U. Sommer and V. A. Baulin, ACS Nano, 2012, 6, 10555-10561.

6 M. Deserno, K. Kremer, H. Paulsen, C. Peter and F. Schmid, in From Single Molecules to Nanoscopically Structured Materials, ed. T. Basche, K. Mullen and M. Schmidt, Springer-Verlag, Berlin, 2014, vol. 260, pp. 237-283.

7 Z. Cournia, T. W. Allen, I. Andricioaei, B. Antonny, D. Baum, G. Brannigan, N.-V. Buchete, J. T. Deckman, L. Delemotte, C. del Val, R. Friedman, P. Gkeka, H.-C. Hege, J. Hénin, M. A. Kasimova, A. Kolocouris, M. L. Klein, S. Khalid, M. J. Lemieux, N. Lindow, M. Roy, J. Selent, M. Tarek, F. Tofoleanu, S. Vanni, S. Urban, D. J. Wales, J. C. Smith and A.-N. Bondar, J. Membr. Biol., 2015, 248, 611-640.

8 G. Rossi and L. Monticelli, Biochim. Biophys. Acta, Biomembr., 2016, 1858, 2380-2389.

9 J. Barnoud, G. Rossi and L. Monticelli, Phys. Rev. Lett., 2014, 112, 068102.

10 H. M. Ding, W. D. Tian and Y. Q. Ma, ACS Nano, 2012, 6, 1230-1238.

11 A. M. Jackson, J. W. Myerson and F. Stellacci, Nat. Mater., 2004, 3, 330-336.

12 A. Verma and F. Stellacci, Small, 2010, 6, 12-21. 
13 A. Verma, O. Uzun, Y. Hu, Y. Hu, H. S. Han, N. Watson, S. Chen, D. J. Irvine and F. Stellacci, Nat. Mater., 2008, 7, 588-595.

14 P. Gkeka, L. Sarkisov and P. Angelikopoulos, J. Phys. Chem. Lett., 2013, 4, 1907-1912.

15 R. C. Van Lehn, P. U. Atukorale, R. P. Carney, Y. S. Yang, F. Stellacci, D. J. Irvine and A. Alexander-Katz, Nano Lett., 2013, 13, 4060-4067.

16 F. Simonelli, D. Bochicchio, R. Ferrando and G. Rossi, J. Phys. Chem. Lett., 2015, 6, 3175-3179.

17 P. Gkeka, P. Angelikopoulos, L. Sarkisov and Z. Cournia, PLoS Comput. Biol., 2014, 10, e1003917.

18 S. Katira, K. K. Mandadapu, S. Vaikuntanathan, B. Smit and D. Chandler, eLife, 2016, 5, e13150.

19 O. G. Mouritsen, Curr. Opin. Colloid Interface Sci., 1998, 3, 78-87.

20 S. Asakura and F. Oosawa, J. Polym. Sci., 1958, 33, 183-192.

21 D. Marenduzzo, K. Finan and P. R. Cook, J. Cell Biol., 2006, 175, 681-686.

22 K. L. Chen and G. D. Bothun, Environ. Sci. Technol., 2014, 48, 873-880.

23 S. Kumar, D. Bouzida, R. H. Swendsen, P. A. Kollman and J. M. Rosenberg, J. Comput. Chem., 1992, 13, 1011-1021.
24 G. M. Torrie and J. P. Valleau, J. Comput. Phys., 1977, 23, 187-199.

$25 \mathrm{H}$. Bouvrais, in Advances in Planar Lipid Bilayers and Liposomes, ed. I. Aleš, Academic Press, 2012, vol. 15, pp. $1-75$.

26 Y. Wang, P. Gkeka, J. E. Fuchs, K. R. Liedl and Z. Cournia, Biochim. Biophys. Acta, Biomembr., 2016, 1858, 2846-2857.

27 J. Yoo and Q. Cui, Biophys. J., 2013, 104, 128-138.

28 S. J. Marrink, H. J. Risselada, S. Yefimov, D. P. Tieleman and A. H. de Vries, J. Phys. Chem. B, 2007, 111, 7812-7824.

29 S. Pronk, S. Pall, R. Schulz, P. Larsson, P. Bjelkmar, R. Apostolov, M. R. Shirts, J. C. Smith, P. M. Kasson, D. van der Spoel, B. Hess and E. Lindahl, Bioinformatics, 2013, 29, 845-854.

30 J. F. Nagle and S. Tristram-Nagle, Biochim. Biophys. Acta, 2000, 1469, 159-195.

31 W. Humphrey, A. Dalke and K. Schulten, J. Mol. Graphics, 1996, 14(33-38), 27-38.

32 H. J. C. Berendsen, J. P. M. Postma, W. F. Vangunsteren, A. Dinola and J. R. Haak, J. Chem. Phys., 1984, 81, 36843690 .

33 G. Lukat, J. Kruger and B. Sommer, J. Chem. Inf. Model., 2013, 53, 2908-2925. 